\title{
Der Restkohlenstoff= und Reststickstoffgehalt des Blutes des Ertrunkenen.
}

\author{
Von \\ Takesi Inouye. \\ (井上 㓮) \\ (Aus dem gerichtlich-medizinischen Institut der Kaiserlichon \\ Universitätzu Sendai. Vorstand:Prof.Dr.T. Ishikawa.)
}

Im Schrifttum auf dem Gebiet der gerichtlichen Medizin liegt eine grosse Anzahl von Arbeiten über das Blut des Ertrunkenen vor, aber sie beschränken sich gewöhnlich auf die Frage, ob die Verdünnung des Blutes eintritt oder nicht, da sie den Zweck verfolgen, diagnostisch verwertbare Merkmale des Ertrinkungstodes zu finden. Soweit ich nachschlagen konnte, sind intravital vorkommene physiologische und chemische Geschehnisse noch nicht Gegenstand einer systematischen Untersuchung gewesen.

In einer früheren Mitteilung über Veränderungen des Restkohlenstoff- und Reststickstoffgehalts des Blutes beim akuten Erstickungstod $^{13}$ wurde gezeigt, dass die dabei eintretende erhebliche Steigerung des Restkohlenstoffs sowie auch des Reststickstoffs nicht unmittelbar vom Sauerstoffmangel herrührt, wie man früher im allgemeinen zu glauben geneigt war, sondern hauptsächlich auf Krämpfe und heftige Bewegungen während der Agonie zurückzuführen ist.

Ertrinken ist selbstverständlich ein Erstickungstod. Aber es verläuft etwas anders als der akute Tod durch Strangulation. Ausserdem treten bekanntlich beim Ertrinken eigenartige Veränderungen der Blutkonzentration ein. Deshalb sei hier als Fortsetzung der genannten Untersuchung das Verhalten des Restkohlenstoffs und -stickstoffs des Blutes beim Ertränken behandelt.

Jüngst hat Spitz ${ }^{2}$ berichtet, dass bei Anoxämie der Restkohlenstoffgehalt des Blutes zunimmt, während der Reststickstoff sich nicht

1) In o uye, T., Tohoku J. Exp. Med., 1934, 24, 100.

2) Spitz, A., Zschr. ges. exp. Med., 1934, 93, 465. 
merklich verändert. Aber die Anordnung seiner Versuche ist grundverschieden von der der meinigen. In der Arbeit Spitzs steht, dass Stepp einen hohen Restkohlenstoffwert in einem unmittelbar nach Erhängen entnommenen Blut gewonnen hat. Aber ich vermisste eine solche Angabe in der von $\mathrm{Spitz}$ angeführten sowie auch in anderen Arbeiten von Stepp. ${ }^{1)} \mathrm{Im}$ häuser $\mathrm{r}^{2}$ hat beim Hund nach Abklemmung der Trachea eine nicht besonders starke Erhöhung des Restkohlenstoffs gefunden. Aber man kann bei ihm nicht erkennen, ob es sich dabei um akuten Erstickungstod handelte, da in seiner Abhandlung eine ausführliche Beschreibung des Versuchs fehlt. Sonst konnte ich keine Angabe über den Restkohlenstoff beim akuten Erstickungstod finden.

In dieser Arbeit wurde das Kaninchen entweder in Süss- oder Salzwasser ertränkt und der Restkohlenstoff- und Reststickstoffgehalt des Blutes vor und unmittelbar oder bestimmte Zeit nach dem Tod des Tieres bestimmt.

Es ergab sich, dass beide Reststoffe des Blutes deutlich stärker zunehmen als bei der Strangulation, ohne Unterschied der Natur des Ertränkungsmediums, wenn man vom stark verdünnten linken Herzblut absieht. Das lässt sich offenbar dadurch erklären, dass das Tier beim Ertränken unter sauerstoffbedürftigen Umständen längere Zeit am Leben bleibt als bei der Strangulation und inzwischen heftige Schwimmbewegungen macht. Dies liefert eine neue Stütze für die Annahme, dass die Steigerung der Reststoffe des Blutes bei Erstikkung im wesentlichen durch Krämpfe und heftige Bewegungen bedingt ist.

Da fast alle früheren Angaben da rin übereinstimmen, dass infolge des Unterschiedes im osmotischen Druck das Blut beim Ertrinken in Süsswasser sich verdünnt und beim Ertrinken im Meer sich verdickt, wurden in dieser Untersuchung Blutproben aus der linken und der rechten Her'zkammer entnommen und vergleichend analysiert. Im Süsswasserversuch wiesen die für den Restkohlenstoff und -stickstoff in beiden Blutarten gefundenen Werte auf die eingetretene Verdünnung des Blutes hin. Im Seewasserversuch konnte man aber, im Gegensatz zu früheren Angaben, kein sichtbares Zeichen der Bluteindik-

1) Stepp, W., Zschr. physiol. Chem., 1916, 97, 213; Dtsch. Arch. klin. Med., 1916, 120, 384 ; ibid, 1918, 124, 177 u. 199; Biochem. Zschr., 1918, 87, 135; Münch. med. Wschr., 1919, 771; Ergebn. Physiol., 1921, 19, 291; Med. klin., 1931, 531 ; S te p p, W. u. S a uer, J., Dtsch. Arch. klin. Med., 1929, 165, 223 u. 232.

2) I m häuser, K., Zschr. ges. exp. Med., 1932, 82, 552. 
Der Restkohlenstoff- und Reststickstoffgehalt des Blutes des Ertrunkenen 493

kung wahrnehmen. Das Ein- und Austreten der Flüssigkeit durch die Lunge scheint nicht einfach durch das osmotischen Druckgefälle verstanden werden zu können.

\section{Material und Methodik.}

Kräftige, junge Kaninchen wurden als Versuchstiere gebraucht und ebenso wie bei meinen früheren Versuchen vorbereitet. Als Kontrolle wurden etwa $4 \mathrm{ccm}$ arteriellen Blutes durch Herzpunktion entnommen, in ein Gefäss mit einer berechneten Fluornatriummenge getan und der Restkohlenstoff- und Reststickstoffgehalt analysiert. Nach über $2 \frac{1}{2}$ Stunden langer Ruhezeit wurde das Tier ertränkt. Zum Ertränken wurde Leitungs- oder künstliches Seewasser, etwa $150 \mathrm{~L}$, in einem Metallbassin von $80.55 .55 \mathrm{~cm}$ verwendet. Das Seewasser wurde durch Auflösen von Rohkochsalz in Leitungswasser im Verhältnis von $3, \tilde{j}: 100$ hergestellt. Um etwaige Einflüsse der Temperatur auf die Zusammensetzung des Blutes auszuschalten, wurde die Temperatur des Wassers stets $2-3^{\circ}$ niedriger als die Körpertemperatur gehalten, ein Wärmegrad, der nach den Angaben von Ima ga w a ${ }^{12}$ und Tad ano und Morim o to $0^{2}$ die thermisch indifferente Badewärme fürs Tier darstellt. Die Kontrollbestimmung wurde mit dem linken Herzblut allein ausgeführt, um eine durch zu grosse Blutentnahme möglicherweise eintretende Beeinträchtigung der Resultate zu verhüten. Ausser im Gehalt an Gasen, mögen sich das Blut im linken Herzen und das im rechten in ihrer. Zusammensetzung unterscheiden, wie insbesondere für den vermuteten Umbau oder Ansatz gewisser fester Blutbestandteile in der Lunge ofters angegeben wird. Jedoch sind die angegebenen Unterschiede gewöhnlich gering und beeinträchtigen nicht die Resultate vorliegender. Untersuchungen.

Die Eltränkung des Tieres wurde in folgender Weise bewerkstelligt. Nachdein an der Vorderbrust des zu ertränkenden Kaninchens ein Gewicht von $70 \mathrm{~g}$, bei einigen Versuchen von $150 \mathrm{~g}$, befestigt worden, wurde das Tier ruhig ins Wasser gelassen. Anfänglich, während einiger Minuten, pflegte das Tier herumzuschwimmen oder versuchte manchmal, an der Seitenwand des Bassins aus dem Wasser herauszukriechen. Es sei hinzugefügt, dass, wenn das Tier eine Pause machen wollte, dasselbe durch einen leichten Handschlag wieder zur Bewe-

1) I magawa, S., Kokumin Eisei, 1929, 6, 1021.

2) Tadano, S. u. Morimoto, K., Ibid, 1933, 10, 933. 
gung getrieben wurde. Das Tier ermattete allmählich und konnte nach einiger Zeit die Nasenöftnung nur noch mit grosser Mühe über Wasser halten. Die Nase begann immer häufiger, länger und tiefer unter Wasser zu kommen. Zu gleicher Zeit wurden die Schwimmbewegungen nach und nach schwächer und blieben später öfters aus, wobei der Kopf sofort ins Wasser sank, bis sie schliesslich, nach 5-6 Minuten, völlig aufhörten. Nun sank das Tier kraftlos auf den Boden des Bassins und geriet in Agonie. Bald darauf tat es den letzten Atemzug.

Nun wurde derHerzschlag auskultiert, wobei die Brust des Tieres aus dem Wasser gehoben wurde, der Kopf aber noch im Wasser blieb. Sobald die Herztöne nicht mehr auskultierbar waren, wurde die Leiche aus dem Bassin herausgenommen, das Herz schnell blossgelegt und ungefähr je $4 \mathrm{ccm}$ Blut beiden Ventrikeln mit Fluorid enthaltenden Spritzen entnommen. Die vom Einbringen des Tieres ins Wasser bis zum Nichtauskultierbarwerden der Herztöne verflossene Zeit wird in dieser Abhandlung als Dauer des Ertrinkens bezeichnet. $\mathrm{Zu}$ erwähnen ist noch, dass vom Aufhören der Herztöne bis zum Zusatz des Enteiweissungsreagens, zu den Blutproben 7-8 Minuten verstrichen.

Die Blutproben wurden möglichst schnell nach Folin und $\mathrm{Wu}$ mit Wolframat enteiweisst und das eiweissfreie Filtrat zur Bestimmung des Restkohlenstoffs und -stickstoffs verwendet, was alles genau wie bei der Untersuchung des Blutes beim Erstickungstod durch Erdrosseln durchgeführt wurde.

\section{Versuche mit Süsswasser.}

Die in diesen Versuchen erhaltenen Zahlen sind in Tabelle I wiedergegeben.

Die Werte für den Restkohlenstoff betrugen also von 170,6 bis $z u$ $200,8 \mathrm{mg}-\%$ vor und von 251,7 bis $333,1 \mathrm{mg}-\%$ für venöses Blut und von 131,1 bis 229,5 mg-\% für arterielles Blut nach dem Ertränken. Der Reststickstoffgehalt, der während des Lebens von 37,7 bis 42,5 mg-\% betrug, stieg nach dem Ertränken auf 41,6 bis $53,2 \mathrm{mg-} \% \mathrm{im}$ venösen und auf $30,8 \mathrm{bis} 43,4 \mathrm{mg}-\%$ im arteriellen Blut.

Das Blut im rechten Ventrikel, das anscheinend weniger verdünnt worden war als das im linken, enthielt bedeutend mehr Reststoffe als bei der Kontrolle. Die Zunahme des Restkohlenstoffs beträgt 60,3 bis $159,3 \mathrm{mg}-\%$, d.h. 30,0 bis $91,7 \%$ des Anfangswerts. Die Zunahme 
Tabelle 1.

Versuche mit Süsswasser.

\begin{tabular}{|c|c|c|c|c|c|c|c|c|}
\hline \multirow{3}{*}{ Kaninchen } & \multirow{3}{*}{ 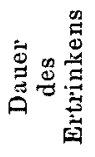 } & \multirow{3}{*}{ 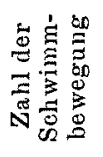 } & \multicolumn{3}{|c|}{ Rest-C (mg-\%) } & \multicolumn{3}{|c|}{ Rest-N (mg-\%) } \\
\hline & & & \multirow{2}{*}{$\begin{array}{c}\text { Anfang- } \\
\text { swert }\end{array}$} & \multicolumn{2}{|c|}{ Sofort nach Tod } & \multirow{2}{*}{$\begin{array}{c}\text { Anfang- } \\
\text { swert }\end{array}$} & \multicolumn{2}{|c|}{ Sofort nach Too } \\
\hline & & & & link. & recht. & & link. & recht. \\
\hline $\mathrm{Nr} .13$ & $7 / 30^{\prime \prime}$ & 125 & 170,6 & 131,1 & 251,7 & 37,8 & 33,9 & 41,6 \\
\hline $\mathrm{Nr} .14$ & $7^{\prime} 00^{\prime \prime}$ & 189 & 175,3 & $\left(\begin{array}{c}-\infty, 0,0) \\
147,4\end{array}\right.$ & $\begin{array}{l}(81,1) \\
259,8\end{array}$ & 39,5 & $\begin{array}{c}(-5,8) \\
30,8\end{array}$ & $\begin{array}{l}(0,8) \\
42,3\end{array}$ \\
\hline $\begin{array}{l}\hat{o} 2,3 \mathrm{~kg} \\
\mathrm{Nr} .15\end{array}$ & $8^{\prime} 30^{\prime \prime}$ & 147 & 200,8 & $\begin{array}{l}(-27,9) \\
146,2\end{array}$ & $\begin{array}{l}(84,5) \\
261,1\end{array}$ & 42,5 & $\begin{array}{c}(-8,7) \\
39,3\end{array}$ & $\begin{array}{l}(2,8) \\
45,5\end{array}$ \\
\hline $\begin{array}{l}\hat{0} 2,2 \mathrm{~kg} \\
\mathrm{Nr} .16\end{array}$ & $5^{\prime} 30^{\prime \prime}$ & 70 & 173,8 & $\begin{array}{l}(-54,6) \\
227,1\end{array}$ & $\begin{array}{l}(60,3) \\
333,1\end{array}$ & 38,0 & $\begin{array}{c}(-3,2) \\
39,4\end{array}$ & $\begin{array}{l}(3,0) \\
46,9\end{array}$ \\
\hline$\hat{\mathrm{o}} 2,1 \mathrm{~kg}$ & & & & $(53,3)$ & $(159,3)$ & & $(1,4)$ & $(8,9)$ \\
\hline $\begin{array}{l}\mathrm{Nr} .17^{*} \\
\hat{0} \quad 1,9 \mathrm{~kg}\end{array}$ & $4^{\prime} 40^{\prime \prime}$ & 47 & 172,2 & $\begin{array}{l}229,5 \\
(57,3)\end{array}$ & $\begin{array}{r}303,6 \\
(131,4)\end{array}$ & 37,7 & $\begin{array}{l}43,4 \\
(5,7)\end{array}$ & $\begin{array}{r}53,2 \\
(15, \tilde{5})\end{array}$ \\
\hline
\end{tabular}

des Reststickstoffs beträgt 2,8 bis $15,5 \mathrm{mg}-\%$, d.h. 7,1 bis $41,1 \%$ des Anfangswerts.

Besonders hoch war die Zunahme bei zwei Fällen, Kaninchen Nr. 16 und 17, auch im Vergleich mit den beim Erdrosseln gefundenen Werten auffallend gross. Die wirklich erfolgte Vermehrung muss bestimmt noch viel grösser gewesen sein, da sich auch bei diesen Fällen das Gesamtblut etwas verdünnt haben muss. Die Zunahme in den übrigen Fällen ist zwar niedriger als in jenen, aber doch so hoch wie die höchsten bei den Erdrosselungsversuchen gefundenen Werte. In diesen drei Fällen war die Verdünnung höchstwahrscheinlich stärker als in anderen, weil die Dauer des Ertrinkens merklich länger war. Man geht wohl nicht fehl in der Behauptung, dass sowohl der Restkohlenstoff wie der Reststickstoff beim Ertränken in weit grösserem Masse als beim Erdrosseln zunimmt. Auch die Ergebnisse der Seewasserversuche sprechen dafür.

Die Ziffern für das arterielle Blut sind sämtlich sehr viel kleiner als die für das venöse. Der Unterschied im Restkohlenstoffwert liegt zwischen 74,1 und $120,6 \mathrm{mg}$ und der im Reststickstoffwert zwischen 6,2 und $11,5 \mathrm{mg}$ pro $100 \mathrm{ccm}$ Blut. Die für die ersten drei Kaninchen gefundenen Werte sind sogar niedriger als die der Kontrolle, wogegen in den zwei letzten Versuchen die Reststoffwerte mehr oder minder gestiegen waren.

Dass sich das Blut beim Ertrinken in Süsswasser nicht unbe- 
deutend verdünnt, ist von zahlreichen Forschern festgestellt worden. In der Mehrzahl der fruheren Untersuchungen wurden gewisse Eigenschaften und Bestandteile des Blutes aus der linken und der rechten Herzkammer miteinander vergleichend bestimmt, statt die vor und nach dem Ertrinken entnommenen Blutproben zu vergleichen. Dabei wurde in erster Linie der osmotische Druck (Kryoskopie) als Mass der Blutkonzentration verwendet, sodann die Trockensubstanz, Hämoglobin, rote Blutkörperchen, spezifisches Gewicht, elektrische Leitfähigkeit, Asche, Chlor, Eisen, weisse Blutkörperchen, Viskosität, Brechungsvermögen, Hämolyse und Eiweiss. Weiter wurde einmal der Gesamtstickstoff zu demselben Zweck benutzt. Manchmal hat man sich so vorgenommen, dass man die Konzentration der Farbstoffe oder anderer, dem Ertränkung'smedium zugesetzten und während des Versuchs durch die Lunge resorbierten Fremdstoffe im arteriellen und venösen Blut zu messen und miteinander zu vergleichen. Das arterielle Blut wurde immer weniger konzentriert als das venöse gefunden. In Übereinstimmung mit den früheren Angaben zeigt das von mir beobachtete eigentümliche Verhalten des Reststickstoffs und besonders des Restkohlenstoffs beim Ertrinken deutlich die erfolgte Verdünnung des Blutes, insbesondere im kleinen Kreislauf.

Die Verdünnung des Blutes beim Ertrinken in Süsswasser berult, wie man allgemein annimmt, auf der Resorption des Wassers durch die Lunge. Es gibt aber, meines Erachtens, noch einen zweiten, wenn auch nicht hauptsächlicher Faktor, welcher aber doch etwas an der Verdünnungserscheinung teilzunehmen scheint. Das ist die Resorption des Wassers vom Verdauungstraktus aus. Ertrinkende Menschen und Tiere verschlucken gewöhnlich reichlich Wasser. Das verschluckte Wasser wird wahrscheinlich während des Lebens noch genügend resorbiert, um auf das Blut mehr oder minder verdunned wirken zu können.

\section{Versuche mit Seewasser.}

Zum Vergleich mit den obigen Versuchen wurde in den Versuchen dieses Abschnittes künstliches Seewasser als Ertränkungsmittel gebraucht. Die Ergebnisse sind in Tabelle 2 zusammengestellt.

Der Restkohlenstoff wie der Reststickstoff zeigten im arteriellen ebenso wie im venösen Blut stets erhebliche Zunahme nach dem Ertränken in Seewasser. Jener stieg von 130,2 bis $156,0 \mathrm{mg}-\%$ auf 211,8 bis $237,9 \mathrm{mg}-\%$ im arteriellen und auf 248,2 bis $268,6 \mathrm{mg} \%$ im ve- 
Tabelle 2.

Versuche mit Seewasser.

\begin{tabular}{|c|c|c|c|c|c|c|c|c|}
\hline \multirow{3}{*}{ Kaninchen } & \multirow{3}{*}{ 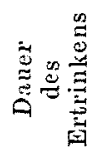 } & \multirow{3}{*}{ 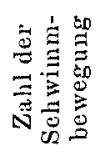 } & \multicolumn{3}{|c|}{ Rest-C (mg-\%) } & \multicolumn{3}{|c|}{ Rest-N (mg-\%) } \\
\hline & & & \multirow{2}{*}{$\begin{array}{c}\text { Anfang- } \\
\text { swert }\end{array}$} & \multicolumn{2}{|c|}{ Sofort nach Tod } & \multirow{2}{*}{$\begin{array}{c}\text { Anfang- } \\
\text { swert }\end{array}$} & \multicolumn{2}{|c|}{ Sofort nach Tod } \\
\hline & & & & link. & recht. & & $\operatorname{lin} k$ & recht. \\
\hline \multirow{8}{*}{$\begin{array}{l}\mathrm{Nr} .18 \\
\delta 2,0 \mathrm{~kg} \\
\mathrm{Nr} .19 \\
\hat{\delta}_{2,0 \mathrm{~kg}} \\
\mathrm{Nr} .20 \\
\hat{\delta} 2,5 \mathrm{~kg} \\
\mathrm{Nr}_{1} .21^{*} \\
\hat{\delta} 1,9 \mathrm{~kg}\end{array}$} & \multirow[t]{2}{*}{$9100^{\prime \prime}$} & \multirow[t]{2}{*}{132} & \multirow[t]{2}{*}{142,1} & 237,9 & 268.6 & \multirow[t]{2}{*}{38,5} & 51,9 & 51,6 \\
\hline & & & & $(95,8)$ & $(120,5)$ & & $(13,4)$ & $(13,1)$ \\
\hline & \multirow[t]{2}{*}{$7 / 10^{\prime \prime}$} & \multirow[t]{2}{*}{136} & \multirow[t]{2}{*}{130,2} & 211,8 & 248,2 & \multirow{2}{*}{30,9} & 33,5 & 33,8 \\
\hline & & & & $(81,6)$ & $(118,0)$ & & $(2,6)$ & $(2,9)$ \\
\hline & \multirow[t]{2}{*}{$9^{\prime} 15^{\prime \prime}$} & \multirow[t]{2}{*}{134} & \multirow[t]{2}{*}{151,2} & 225,1 & 252,9 & \multirow[t]{2}{*}{36,5} & 42,2 & 44,6 \\
\hline & & & & $(73,9)$ & $(101, \pi)$ & & $(5,7)$ & $(8,1)$ \\
\hline & \multirow[t]{2}{*}{$9^{\prime} 00^{\prime \prime}$} & \multirow[t]{2}{*}{101} & \multirow{2}{*}{156,0} & 237,0 & 267,1 & \multirow[t]{2}{*}{40,1} & 47,9 & 49,7 \\
\hline & & & & $(81,0)$ & $(111,1)$ & & $(7,8)$ & $(9,6)$ \\
\hline
\end{tabular}

* Ein viertel Stunden nach dem Tod bearbeitet.

Die eingeklammerten Zahlen bedenten die Zunahme.

nösen Blut und dieser von 30,9 bis $40,1 \mathrm{mg}-\%$ auf 33,5 bis $51,9 \mathrm{mg}-\%$ im arteriellen und auf 33,8 bis $51,6 \mathrm{mg}-\%$ im venösen Blut. Die Zunahme im Blut des rechten Ventrikels beträgt für den Restkohlenstoff 67,3 bis $90,6 \%$ und für den Reststickstoff 9,4 bis $34,0 \%$ des Anfangswertes. DieZahlen fürs Blut des linken Ventrikels betragen 48,9 bis $67,4 \%$ und 8,4 bis $34,8 \%$. Diese Ziffern sind grösser als in den Versuchen mit Süsswasser sowie beim Erdrosseln.

Die hohen Reststoff werte beruhen zum Teil darauf, dass die Dauer des Ertrinkens hier im allgemeinen länger als in den Süsswasserversuchen war, aber der Hauptsache nach auf dem Verdünnungsverlältnis. Nach der allgemeinen Vorstellung verdünnt sich das Blut beim Ertrinken in Süsswasser, aber beim Ertrinken in Seewasser verdickt es sich. Bevor man die Stärke der Reststoffzunahme schätzt, muss die Frage beantwortet werden, ob und wieweit das Blut in unseren Versuchen verdickt worden ist.

Das Schrifttum über die Verschiebung der Blutkonzentration beim Ertrinken in Seewasser ist nicht so umfangreich wie das für das Ertrinken in Sïsswasser. Carrara $a^{1)}$ bestimmte dazu die Gefrierpunktserniedrigung und Leitfähigkeit neben Hämoglobin, Wasser und Erythrozyten des arteriellen und venösen Blutes beim in Seewasser ertränkten Hunde. Stoenescu ${ }^{2}$ bediente sich ebenfalls der Gefrier-

1) Carrara. MI., Folia haemat., 1904, 1, 330; Vischr. ger. Med., 1902, 3. F. 24, 236.

2) Stoenescu, N., Ann. d'Hyg. Publ. et Héd. Lég., 1903, 3. Sér. 49, 14. 
punktserniedrigung, Gettler ${ }^{12}$ und $\mathrm{Yamakami} \mathrm{i}^{2}$ verwandten den Chlorgehalt als Mass der Konzentration des Blutes, Szulislawska

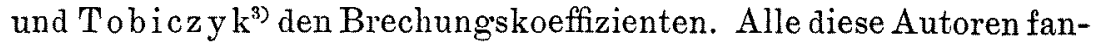
den übereinstimmend das Blut im linken Ventrikel stärker konzentriert als das im rechten. Dies ist nach ihnen die Folge des Austrittes des Wassers aus dem Blut in den osmotisch konzentrierteren Alveolarinhalt.

Eine Ausnahme bildet eine Arbeit von Nakai,4) welcher den Gesamtstickstoff im Blut oder Koagulum aus dem rechten Herzen stets, sowohl in Süss- wie auch in Seewasser, in grösseren Mengen als in dem aus dem linken fand, nur dass beim letzten die Unterschiede zwischen den beiden Blutarten weniger ausgeprägt waren. In seiner Arbeit wurden die Proben bei einem Kaninchen sofort und bei den übrigen Tieren, Kaninchen und Hunden, einige Tage nach dem Ertränken entnommen. Er ist der Meinung, dass die Blutverdünnung ohne Rücksicht auf die Natur der Ertränkungsflüssigkeit eintritt.

In der vorliegenden Untersuchung wurden die Reststoffe, im Gegensatz zur jetzt allgemein vertretenen Ansicht, im Blut des linken Ventrikels nicht höher, sondern eher etwas niedriger als im rechten gefunden. Das arterielle Blut enthielt nämlich 211,8 bis $237,9 \mathrm{mg}-$ $\%$ Restkohlenstoff und 33,5 bis $51,9 \mathrm{mg}-\%$ Reststickstoff und das venöse 248,2 bis $268,6 \mathrm{mg}-\%$ und 33,8 bis $51,6 \mathrm{mg}-\%$. Allerdings liegen die Unterschiede des Reststickstoffs innerhalb der Grenzen der Versuchsfehler. Dafür scheinen allerdings teilweise seine, im Vergleich zu den andren, eigentlich viel kleineren Werte verantwortlich zu sein, jedenfalls liegen die Werte für Venenblut höher als für Arterienblut, mit nur einer Ausnahme, Nr. 18.

In üblicher Weise beurteilt, muss man sagen, dass auch Seewasser das Blut des Ertrinkenden verdünnend beeinflusst. Aber etwaige Unterschiede in der Konzentration irgendeiner Substanz oder in gewissen Eigenschaften beider Blutarten sind nicht notwendigerweise als endgültige Beweise erfolgter Konzentrationsveränderungen zu betrachten, wie man allgemein annimmt. Die Eigenschaften und die Bestandteile des Blutes können sich unter verschiedenen Verhältnissen verändern. Über den osmotischen Druck z.B. gibt es im neueren

1) Gettler, A. O., J. Amer. Med. Assoc., 1921, 77, 1650.

2) Yamaka mi, K., Tohoku J. Exp. Med, 1923-24, 4, 88 .

3) Szulislawska u. Tobiczyk, Dtsch. Zschr. ger. Med., 1927, 9, 13.

4) N akai, R., Arb. med. Univ. Okayama, 1930, 2, 63. 
Schrifttum Arbeiten von Häbler und Weber ${ }^{12}$ und Hinsberg und S ch ürme y er, ${ }^{2)}$ nach welchen die Gefrierpunktserniedrigung von der $\mathrm{CO}_{2}$-Spannung des Blutes abhängt. $\mathrm{Kar} \cdot \mathrm{g}^{\mathrm{e}} \mathrm{r}^{33}$ machte darauf aufmerksam, dass der Chlorgehalt in den drei Blutsystemen, dem arteriellen, kapillaren und venösen, ziemlich bedeutend voneinander abweichen können. In einem Vortrag Revens tor $\mathrm{fs}^{4)}$ findet man folgende Worte: „Lungenödem infolge vorzeitiger Erlähmung des linken Ventrikels bei fortarbeitendem rechten Herzen müsste in ausgesprochenen Fällen erhebliche Unterschiede in der Zusammensetzung des Blutes beider Herzhälften herbeiführen". Er gab ferner an, dass fast in jeder 2448 Stunden alten Leiche das Blut in beiden Herzhälften verschieden konzentriert ist, da die Geschwindigkeit der postmortal eintretenden Eindickung für das linke und das rechte Herzblut nicht gleich ist.

In unseren Fällen lassen sich die bei beiden Blutarten gefundenen kleinen Unterschiede möglicherweise auch dadurch erklären, dass sich von Organen und Geweben herrührende Reststoffe als Folge der ziemlich schnell abnehmenden Herztätikeit im venösen System etwas stärker angehäuft hatten. Mit anderen Worten, sie sind die Folge des gerade umgekehrt wie bei der stärkeren Verdünnung des linken Herzblutes wirkenden Mechanismus. Anderscits aber darf man nicht ohne weiteres Blutverdünnung ausschliessen, weil für das rechte Herzblut die Zunahme kleiner als in den Süsswasserversuchen mit kürzerer Dauer des Ertrinkens war. Ich verzichte vorläufig darauf, etwas Bestimmtes darüber zu sagen, bis weitere Daten gewonnen werden. Aber soviel ist jetzt schon sicher, beim Ertrinken in Seewasser verdickt sich das Blut nicht oder wenigstens nicht so bedeutend, wie man allgemein annimmt. Dass zwischen beiden Blutarten kein grosser Unterschied besteht, stellt die Eigentümlichkeit des Ertrinkens in Seewasser dar und scheint darauf hinzudeuten, dass sich die Blutkonzentration in den Lungenkapillaren nicht nennenswert verändert hat.

Nun scheint diese Tatsache gegen die allgemeine Vor'stellung zu sprechen, dass die Veränderung der Blutkonzentration beim Ertrinken eine reine osmotische Erscheinung sei, da die Flüssigkeit beim Süsswasser ins Blut einfliesst und beim Seewasser aus dem Blut ausfliesst. Wäre die Alveolarwandung zusammen mit der Kapillarwand einfach

1) Häbler, C. u. We ber, K., Biochem. Zschr., 1928, 195, 364.

2) Hinsberg, K. u. Schürmeyer, A., Zschr. ges. exp. Med., 1932, 82, 696.

3) Karger, K., Klin. Wsehr., 1927, 6, 1994.

4) Revenstorf, Vjschr. ger. Med., 1907, 3. F. 33, Suppl., 50. 
eine richtige osmotische Membran, so würde das Blut, wie man meint, beim Ertrinken in Seewasser eine ansehnliche Menge Wasser verlieren, da das osmotische Gefälle zwischen Seewasser und Blut ungefähr dreimal so gross, und zwar mit entgegengesetztem Vorzeichen, wie zwischen Süsswasser und Blut ist. Aber das war nicht der Fall. Es liegt nahe anzunehmen, dass die Resorption durch die Lunge nicht rein mechanisch durch Diffusion bedingt ist. Das kann man auch aus den Versuchen La que urs ${ }^{1)}$ iber Lungenödem mit Intratrachealinjektion ersehen. Einerseits wurden isotonische Lösungen ebenso schnell wie reines Wasser resorbiert, anderseits wurden auch bei Injektion hypertonischer Lösungen von Glukose und Kochsalz manchmal Ergebnisse erhalten, welche einer erfolgten Resorption gleichkommen. Rein mechanische Vorgänge wie Diffusion oder Filtration genügen daher nicht zur Erklärung des Ein- und Ausströmens des Wassers durch die Lunge. Damit soll aber nicht gesagt sein, dass der osmotische Vorgang dabei keine grosse Rolle spielt. Die Alveolarwandung zeigt anscheinend eine aktive und selektive Resorptionsfähigkeit gegen Wasser und darin gelösten Substanzen und lässt das Wasser leichter nach innen als nach aussen durch. In dieser Hinsicht verhält sich also die Alveolarwand etwa wie die Darmwand oder die Hautdecke. Nebenbei sei bemerkt, es erscheint nicht unmöglich, dass die Alveolarwandung, wie etwa in der Sekretionstheorie von Bohr und anderen, eine spezifische Durchlässigkeit auch für Gase besitzt, mag auch heute der Respirationsgaswechscl im allgemeinen für ein rein physikalischer Diffusionsvorgang gelialten werden.

\section{Ertränkungsversuche mit Heparininjektion.}

In diesem Abschnitt wurde versucht, die postmortalen Veränderungen beim Ertrinken etwas kennen zu lernen. Das Blut des Tieres gerinnt ziemlich schnell selbst nach dem Ertränken, wie Sardas ${ }^{2)}$ eingehende Untersuchung zeigt. Das Blut unseres Kaninchens gerann in der Regel auch in Süsswasser in einer halben Stunde. Deshalb habe ich mich wieder des Heparins bedient, das mir schon bei der Untersuchung über Erdrosseln gute Dienst geleistet hatte. Kurz bevor das Versuchstiel: ins Wasser getan wurde, injizierte ich ihm intravenös $75 \mathrm{mg}$ Heparin. Der Tierleib warde eine Stunde nach dem

1) Laque ur, E., Arch. ges. Physiol., 1920, 184, 104; Laquenu, E. u. D. de Vries Reilingh, Dtsch. Arch. klin. Med., 1920, 131, 310.

2) S a rda, G., Ann. d'Hgg. Publ, et Méd. Lég., 1903, 3. Sér. 49, 125. 
Tod aus dem Wasser herausgenommen, und dann wurden wie üblich Blutproben aus beiden Kammern entnommen. Die Probeentnahme durfte nicht noch weiter verschoben werden, denn das linke Herz war öfters blutleer, wenn es eine bis zu zwei Stunden nach dem Tod geöffnet wurde. Das kommt von der Kontraktion infolge der Totenstarre. Bei unseren Tieren trat ausgeprägte Totenstarre gewöhnlich schon nach einer Stunde ein. Tabelle 3 enthält die Ergebnisse.

\section{Tabelle 3.}

Ertränkungsversuche mit Heparininjektion.

\begin{tabular}{|c|c|c|c|c|c|c|c|c|}
\hline \multirow{3}{*}{ Kaninchen } & \multirow{3}{*}{ 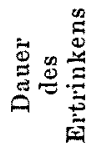 } & \multirow{3}{*}{ 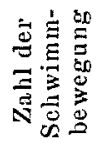 } & \multicolumn{3}{|c|}{ Rest-C (mg-\%) } & \multicolumn{3}{|c|}{ Rest-N (mg-\%) } \\
\hline & & & \multirow{2}{*}{$\begin{array}{c}\text { Anfang- } \\
\text { swert }\end{array}$} & \multicolumn{2}{|c|}{ Sofort nach Tod } & \multirow{2}{*}{$\begin{array}{c}\text { Anfang- } \\
\text { swert }\end{array}$} & \multicolumn{2}{|c|}{ Sofort nach Tod } \\
\hline & & & & link. & recht. & & link. & recht. \\
\hline \multicolumn{9}{|c|}{ Sitss wasser } \\
\hline $\begin{array}{l}\text { Nr. } 22 \\
\hat{o} 2,3 \mathrm{~kg} \\
\mathrm{Nr} \cdot 23 \\
\hat{0} \quad 2,2 \mathrm{~kg}\end{array}$ & $\begin{array}{l}8^{\prime} 30^{\prime \prime} \\
7^{\prime} 40^{\prime \prime}\end{array}$ & $\begin{array}{r}169 \\
169\end{array}$ & $\begin{array}{l}155,2 \\
155,5\end{array}$ & $\begin{array}{l}176,1 \\
(20.9) \\
195,6 \\
(40,1)\end{array}$ & $\begin{array}{r}353,2 \\
(198,0) \\
373,3 \\
(217,8)\end{array}$ & $\begin{array}{l}36,8 \\
37,8\end{array}$ & $\begin{array}{c}32,5 \\
(-4,3) \\
38,9 \\
(1,1)\end{array}$ & $\begin{array}{r}44.3 \\
(7,5) \\
52,6 \\
(14,8)\end{array}$ \\
\hline \multicolumn{9}{|c|}{ Seewasser } \\
\hline $\begin{array}{c}\mathrm{Nr} .24 \\
\text { o } 2,1 \mathrm{~kg} \\
\mathrm{Nr} .25 \\
\text { o } 2,3 \mathrm{~kg}\end{array}$ & $\begin{array}{l}6^{\prime} 15 " 1 \\
7^{\prime} 00^{\prime \prime}\end{array}$ & $\begin{array}{l}110 \\
145\end{array}$ & $\begin{array}{l}138,8 \\
143,4\end{array}$ & $\begin{array}{r}205,9 \\
(67,1) \\
273,1 \\
(129,7)\end{array}$ & $\begin{array}{r}267,9 \\
(129,1) \\
355,3 \\
(211,9)\end{array}$ & $\begin{array}{l}38,1 \\
38,7\end{array}$ & $\begin{array}{l}43,8 \\
(5,7) \\
47,0 \\
(8,3)\end{array}$ & $\begin{array}{r}47,0 \\
(8,9) \\
62,2 \\
(23,5)\end{array}$ \\
\hline
\end{tabular}

Die eingeklammerten Zahlen bedeuten die Zunahme.

Die Werte für beide Reststoffe nach Ertränken in Süsswasser waren wieder im rechten Herzblut höher als im linken, wobei die Unterschiede grösser als im einfachen Süsswasserversuch waren. Es liesse sich vielleicht denken, dass das Blut nach dem Tod noch weiter verdünnt worden war. Damit aber stelit es nicht im Einklang, dass die Werte für das linke Herzblut nicht kleiner, sondern grösser als bei den vorangehenden Versuchen waren.

In diesem Abschnitt wurden überhaupt bei beiden Wässern höhere Reststoffwerte gewonnen. Am stärksten fallen die hoher Ziffern beim Süsswasserversuch in die Augen, bei welchem gerade eine ansehnliche Blutverdünnung zu erwarten war. Es steht ausser Zweifel, dass die Blutverdünnung nicht lange nach dem Tod eintritt. Im Gegenteil, hier verdickte sich postmortal das Blut schon innerhalb einer Stunde. 


\section{Zusammenfassung.}

1. Als Fortsetzung der Arbeit über den Restkohlenstoff und -stickstoff im Blut des Erdrosselten wurde das Blut des Ertrunkenen untersucht. Die Bestimmungen wurden wie früher unmittelbar oder eine bestimmte Zeit nach dem Ertränken des Kaninchens in Süss- und Seewasser ausgeführt.

2. Der Restkohlenstoff ebenso wie der Reststickstoff im rechten Herzblut zeigten erhebliche Erhöhung beim Ertränken sowohl in Süss- als auch in Seewasser. Die Zunahme war im allgemeinen grösser als beim Erdrosseln. Sie betrug für den Restkohlenstoff von 30 bis $92 \%$ in Süsswasser und von 67 bis $91 \%$ in Seewasser und für den Reststickstoff von 7 bis $41 \%$ und von 8 bis $35 \%$ des Anfangswertes.

3. Die Reststoffwerte für das linke Herzblut stiegen beim Ertränken in Süsswasser weit weniger, in den Fällen mit längerer Dauer des Ertrinkens nahmen sie sogar deutlich ab. Das ist die Folge der Blutverdünnung, besonders im linken Herzen, beim Ertrinken in Süsswasser.

4. An der Verdünnung des Blutes scheint sich das geschluckte und in den Verdauungskanal gelangte Wasser etwas zu beteiligen.

5. Dass beim rechten Herzblut, das, wenn auch in geringerem Grad, gleichfalls hätte verdünnt sein müssen, höhere Werte als beim Erdrosseln gefunden wurden, beruht gewiss darauf, dass hier heftige Bewegungen und Krämpfe länger fortdauerten. Dies spricht für die Annahme, dass die Zunahme der Reststoffe beim Erstickungstod wesentlich durch Bewegungen und Krämpfe bedingt ist.

6. Beim Ertrinken in Süsswasser beeinflusst in erster Linie die Dauer des Ertrinkens einerseits den Grad der Blutverdünnung und anderseits die Zunahme der Reststoffwerte. Die Konzentration der Reststoffe im Blut des Ertrunkenen wird durch das dabei herrschende Verhältnis zwischen diesen zwei entgegengesetzt wirkenden Faktoren bestimmt.

7. Auch beim Ertränken in Seewasser wurden im linken Herzblut weniger Reststoffe als im rechten gefunden.

8. Man könnte zunächst daran denken, dass auch beim Ertränken in Seewasser das Blut verdünnt wurde. Dafür waren aber die Unterschiede zwischen den beiden Blutarten zu klein.

9. Dies spricht jedenfalls gegen die allgemein vertretene Ansicht, dass beim Ertrinken in Seewasser das Blut, insbesondere des 
Der Restkohlenstoff- und Reststickstoffgehalt des Blutes des Ertrunkenen 503

linken Herzens, verdickt wird, da die molekulare Konzentration des Seewassers viel grösser als die des Blutes ist.

10. Die Resorption der Flüssigkeiten durch die Lunge scheint nicht einfach durch Diffusion erklärt werden zu können. Die Alveolarwandung besitzt anscheinend eine aktive, beschränkte Resorptionsfähigkeit.

11. Bei den Versuchen, in welchen die Blutgerinnung durch Heparininjektion gehemmt und das Blut eine Stunde nach dem Tod entnommen wurde, war schon postmortale Bluteindickung wahrzunehmen. 